of (9) and (10). Since $G(z, w)$ is continuous in $z$ and $w$ for $w$ on $I_{1}$ or $I_{2}$ and $z$ in $D$ and is a regular function of $z$ in $D$ for a fixed $w$ on $I_{1}$ or $I_{2}$, it results that $J(z, B)$ and $J(z,-B)$ define regular functions in $D$.

Since $D$ contains an interval of the positive real axis for which (8) holds it follows that $J(z, B)+J(z,-B)$ provides the analytic continuation of

$$
f(z)-\sum_{n=1}^{\left[h_{1}\right]} a(n)(-1)^{n} C_{z-1, n}
$$

over the region $D$.

As the region $D$ can be chosen to contain any bounded region of the complex plane in its interior, the theorem follows.

UNIVERSITY OF KENTUCKY

\title{
NON-NEGATIVE OBSERVABLES ARE SQUARES
}

\section{S. SHERMAN}

For $[\mathrm{S}],{ }^{1}$ whose notation and results we use without further comment, the question of whether or not a non-negative observable is the square of an observable is left open. Our positive solution of this question eliminates one of the hypotheses in a pair of propositions [S,Theorem 4 and Corollary 4.1 ] in that paperand so now we know that without exception a pure state of a subsystem may be extended to a pure state of the whole system of observables and that the spectral value of an observable equals the value of the observable in some pure state of the system. This serves to shrink the gap between $\mathcal{S}$, the real linear space of self-adjoint elements of a $C^{*}$ algebra, and $\mathfrak{A}$, a system of observables. Although every $\mathcal{S}$ is isomorphic to an $\mathfrak{A}$, no examples of an $\mathfrak{A}$ not isomorphic to an $\mathcal{S}$ have appeared in the literature, so the gap may be just an apparent one.

THEOREM. In a system of observables the sum of squares of observables is the square of an observable.

Proof. Let $V=\sum_{\alpha=1}^{n} U_{\alpha}^{2}$. By [S, Corollary 1.1] the closed system $\mathfrak{A}(V)$ generated by $V$ (and $I$ ) is isomorphic algebraically and metrically to $C(X, R)$, the collection of real-valued continuous functions on compact Hausdorff space $X$, where $V \leftrightarrow\left(\phi_{V} \mid X\right) \in C(X, R)$ and $\|V\|$

Presented to the Society, December 29, 1949; received by the editors October 26, 1949.

1 [S] refers to the bibliography at the end of the paper. 
$=\sup \left\{\left|\phi_{V}(\omega)\right| \mid \omega \in X\right\}$. Since $\phi_{U}(\omega) \geqq 0$ for each $\omega \in X$, there is a $U^{\prime} \in \mathfrak{Y}(V)$ such that $\phi_{U}(\omega)=\left(\phi_{U},(\omega)\right)^{2}$. In that case $U=\left(U^{\prime}\right)^{2}$. We see that $\left\{U \mid \phi_{U}(\omega) \geqq 0\right.$ for each $\left.\omega \in X\right\}$ is contained in the set of squares of observables. It is sufficient to show that $\phi_{V}(\omega) \geqq 0$ for each $\omega \in X$.

Suppose there exists an $\omega_{0} \in X$ such that $\phi_{V}\left(\omega_{0}\right)<0$. If $\phi_{V}(\omega) \leqq 0$ for each $\omega \in X$, then $-V \geqq 0$, which implies $V=0$ and contradicts the assumption. We are left with the case where $\phi_{V}(\omega)$ assumes both positive and negative values. Let us assume that

$$
\phi_{V}\left(\omega_{0}\right)=\inf \left\{\phi_{V}(\omega) \mid \omega \in X\right\}<0
$$

and

$$
\phi_{V}\left(\omega_{1}\right)=\sup \left\{\phi_{V}(\omega) \mid \omega \in X\right\}>0 .
$$

We seek a contradiction in this. Let

$$
\begin{aligned}
& F(3 / 4-)=\left\{\omega \mid \phi_{V}(\omega) \leqq 3 \phi_{V}\left(\omega_{0}\right) / 4\right\}, \\
& F(1 / 2+)=\left\{\omega \mid \phi_{V}(\omega) \geqq \phi_{V}\left(\omega_{0}\right) / 2\right\} .
\end{aligned}
$$

By applying Tietze's Theorem, we find a $V_{1} \in \mathfrak{X}(V)$ such that

$$
\phi_{V_{1}}(\omega)\left\{\begin{array}{l}
=0, \omega \in F(1 / 2+), \\
=-1, \omega \in F(3 / 4-), \\
\text { lies between } 0 \text { and }-1 \text { for all other } \omega \in X .
\end{array}\right.
$$

It follows that $V_{1} \leqq 0$ and $\left\|V_{1}\right\|=1$. Let

$$
\epsilon=\min \left\{-\phi_{V}\left(\omega_{0}\right)\left(4 \phi_{V}\left(\omega_{1}\right)\right)^{-1}, 2^{-1}\right\}>0 .
$$

If $\omega \in F(1 / 2+)^{\prime}$ (the complement of $F(1 / 2+)$ ), then

$$
\phi_{V_{1}}(\omega)+(1-\epsilon) \geqq-1+(1-\epsilon)=-\epsilon \geqq \frac{\phi_{V}\left(\omega_{0}\right)}{4 \phi_{V}\left(\omega_{1}\right)} \geqq \frac{\phi_{V}(\omega)}{2 \phi_{V}\left(\omega_{1}\right)} .
$$

If $\omega \in F(1 / 2+)$, then

$$
\phi_{V_{1}}(\omega)+(1-\epsilon) \geqq 1-\epsilon \geqq \frac{1}{2} \geqq \frac{\phi_{V}(\omega)}{2 \phi_{V}\left(\omega_{1}\right)} .
$$

Thus

$$
V_{1}+(1-\epsilon) I \geqq V\left(2 \phi_{V}\left(\omega_{1}\right)\right)^{-1} \geqq 0
$$

and

$$
0 \geqq V_{1} \geqq(\epsilon-1) I .
$$


By [S, Postulate II, 4]

$$
\left\|-V_{1}\right\|=\left\|V_{1}\right\| \leq|\epsilon-1|<1
$$

which contradicts our previous assertion that $\left\|V_{1}\right\|=1$. We have found our contradiction and conclude that $\phi_{V}(\omega) \geqq 0$ for each $\omega \in X$ and there exists a $W \in \mathfrak{A}(V)$ such that $V=W^{2}$.

COROLlaRy. The (closed) subsystem of a system generated by an observable is isomorphic, algebraically, metrically, and order-wise, with the system $C(X, R)$ of all real-valued continuous functions on a compact Hausdorff space, where for $\phi_{1}, \phi_{2} \in C(X, R), \phi_{1} \leqq \phi_{2}$ means $\phi_{1}(\omega) \leqq \phi_{2}(\omega)$ for each $\omega \in X$.

(See [S, Corollary 1.1].)

CoRollary. [S, Theorem 4] A pure state of a subsystem coincides on that subsystem with a pure state of the system.

CoRollary. [S, Corollary 4.1] A spectral value of an observable equals the value of the observable in some pure state of the system.

\section{BIBLIOGRAPHY}

[S] I. E. Segal, Postulates for general quantum mechanics, Ann. of Math. vol. 48 (1947) pp. 930-948.

INSTITUTE for Advanced Study 